Research Paper

\title{
Impact of Statin Use on Outcomes in Triple Negative Breast Cancer
}

\author{
Simona F. Shaitelman ${ }^{1}$, Michael C. Stauder ${ }^{1}$, Pamela Allen¹, Sangeetha Reddy², Susan Lakoski³, Bradley \\ Atkinson ${ }^{4}$, Jay Reddy ${ }^{1}$, Diana Amaya ${ }^{1}$, William Guerra ${ }^{1}$, Naoto Ueno ${ }^{2}$, Abigail Caudle ${ }^{4}$, Welela Tereffe ${ }^{1}$, \\ Wendy A. Woodward ${ }^{1 凶}$ \\ 1. Department of Radiation Oncology, University of Texas MD Anderson Cancer Center, Houston, TX \\ 2. Department of Breast Medical Oncology, University of Texas MD Anderson Cancer Center, Houston, TX \\ 3. Department of Clinical Cancer Prevention, University of Texas MD Anderson Cancer Center, Houston, TX \\ 4. Department of Breast Surgical Oncology, University of Texas MD Anderson Cancer Center, Houston, TX \\ $\square$ Corresponding author: Wendy A. Woodward, Associate Professor, Department of Radiation Oncology, University of Texas MD Anderson Cancer Center. \\ 1515 Holcombe Blvd, Unit 1422, Houston, TX 77030. Phone: 713-563-2300; Fax: 713-404-5797; Email: wwoodward@mdanderson.org \\ (c) Ivyspring International Publisher. This is an open access article distributed under the terms of the Creative Commons Attribution (CC BY-NC) license \\ (https://creativecommons.org/licenses/by-nc/4.0/). See http://ivyspring.com/terms for full terms and conditions.
}

Received: 2016.12.13; Accepted: 2017.04.01; Published: 2017.07.05

\begin{abstract}
Purpose: We sought to investigate if the use of HMG Co-A reductase inhibitors (statins) has an impact on outcomes among patients with triple negative breast cancer (TNBC).

Methods: We reviewed the cases of women with invasive, non-metastatic TNBC, diagnosed 1997-2012. Clinical outcomes were compared based on statin use (defined as ever use during treatment vs. never use). We identified a subset of women for whom a 5 -value lipid panel (5VLP) was available, including total cholesterol, low density lipoprotein, high density lipoprotein, very low density lipoprotein, and triglycerides. The Kaplan-Meier method was used to estimate median overall survival (OS), distant metastases-free survival (DMFS), and local-regional recurrence-free survival (LRRFS). A Cox proportional hazards regression model was used to test the statistical significance of prognostic factors.
\end{abstract}

Results: 869 women were identified who met inclusion criteria, with a median follow-up time of 75.1 months (range 2.4-228.9 months). 293 (33.7\%) patients used statins and 368 (42.3\%) had a 5 VLP. OS, DMFS, and LRRFS were not significant based on statin use or type. Controlling for the 5 VLP values, on multivariable analysis, statin use was significantly associated with OS (HR $0.10,95 \%$ $\mathrm{Cl} 0.01-0.76$ ), but not with DMFS (HR $0.14,95 \% \mathrm{Cl} 0.01-1.40$ ) nor LRRFS (HR $0.1095 \% \mathrm{Cl}$ 0.00-3.51).

Conclusions: Statin use among patients with TNBC is not associated with improved OS, although it may have a benefit for a subset of patients. Prospective assessment would be valuable to better assess the potential complex correlation between clinical outcome, lipid levels, and statin use.

Key words: breast cancer, triple negative, statin, cholesterol

\section{Introduction}

Triple negative breast cancers (TNBCs) make up approximately $15 \%$ of all new breast cancer diagnoses annually [1]. TNBCs have been shown to result in worse survival outcomes due to a high rate of distant metastases, and limited targeted therapies are available [2]. Within TNBCs, a heterogeneous group of molecular classifications have been identified, with distinct biological pathways that may serve as differential targets for therapy [3]. Efforts have been made to identify existing, relatively low cost and low toxicity medications that can be repurposed for their anti-cancer effects in TNBC $[4,5]$. The HMG-CoA reductase inhibitors, statins, are cholesterol lowering medications that have been identified as possible agents to prevent cancer growth.

Numerous studies have implicated cholesterol 
regulation in both the risk and progression of breast cancer [6]. Our lab and others have shown that cholesterol regulation can influence pre-clinical models of triple negative breast cancer through a PI3K/FOXO3a pathway, affecting stem cells and metastases [7-9].

However, clinical data on outcomes of breast cancer patients based on statin use have been mixed [10-14], suggesting that the interaction of statins with breast cancer stem cells may be complex and not yet adequately examined. We recently demonstrated that statins influenced disease free survival for inflammatory breast cancer (IBC) patients [15], a disease enriched in triple negative breast cancer. However, in a follow-up retrospective study of IBC patients who received neoadjuvant chemotherapy, surgery and post-mastectomy radiation, we found the statin effect was lost on multivariate analysis when controlling for lipid panels in patients with available data [16]. Based on these data, we hypothesized that in a large cohort of non-metastatic triple negative breast cancer patients, statin association on cancer outcomes could be elucidated. We sought to examine outcomes of patients with TNBC, a more virulent form of breast cancer for which novel therapeutic interventions are needed, based on statin use and the interaction of statin use with other clinical factors including serum lipid levels. Results from this study would be informative for future clinical trial design for this subset of high risk breast cancer.

\section{Methods}

We reviewed the cases of women with newly diagnosed invasive, non-metastatic TNBC treated at the MD Anderson Cancer Center (MDACC), diagnosed between 1997 and 2012 maintained in a prospectively collected database. Patients were defined as statin users if they were documented as taking statins during any of the medical visit encounters at our institution, which occurred anytime between initial diagnosis, during oncologic treatment, or at follow-up. Statins were categorized by lipophilic or hydrophilic status based on the classification outlined by Schachter [17].

Estrogen receptor (ER) and progesterone receptor (PR) status was determined using immunohistochemistry (IHC), with a cutoff of less than $10 \%$ for negativity. Her2 Neu status was considered negative if either: 1) IHC was 0 to $1+$ without fluorescence in situ hybridization (FISH) or 2) FISH results negative. Clinical data collected included: age at diagnosis, menopausal status, race, body mass index at diagnosis, clinical and pathological stage, use and sequencing of chemotherapy, type of definitive surgery, and use of radiation therapy. Where available in our medical record, we recorded results from a 5-value lipid panel, including total cholesterol, low density lipoprotein (LDL), high density lipoprotein (HDL), very low density lipoprotein (VLDL), and triglycerides.

The Institutional Review Board of MDACC approved a protocol for conduct of this study and granted a waiver of informed consent, due to the observational nature of the study.

The primary outcome of this study was overall survival (OS) in years between the date of diagnosis to the date of death or the date of last follow-up. Secondary outcomes included disease free survival (DFS), distant metastases-free survival (DMFS) and local-regional recurrence-free survival (LRRFS). Clinical outcomes were compared based on any statin use (ever use vs. never use) and by type of statins used (hydrophilic, lipophilic, or both).

Clinical variables of interest were summarized using standard descriptive statistics and frequency tables. Fisher's exact test and chi-square tests, as appropriate, were used to determine associations between clinical characteristics. The Wilcoxon rank sum test was used to determine differences in 5-value lipid panel results between statin users and statin non-users. The Kaplan-Meier method was used to estimate median OS, DMFS, and LRRFS.

Univariate Cox proportional hazards regression models were used to test the statistical significance of potential prognostic factors on OS, DM, and LRR. This analysis was performed for the overall cohort and also for the subset of patients with a 5-value lipid panel, in order to control for these values as potential confounders. A Cox multivariable model was created including those clinicopathological factors that remained statistically significant were kept in the model. When available, values for total cholesterol, HDL cholesterol, LDL cholesterol, VLDL cholesterol, and triglycerides were included. Statistical calculations were carried out using Stata/MP 14.1 (Stata Corp 2015, College Station, TX).

\section{Results}

A total of 869 patients with invasive, non-metastatic TNBC were identified, of whom 293 $(33.7 \%)$ had documented usage of statins at some point between breast cancer diagnosis and last oncologic follow-up. Of these patients, 182 (62.1\%) used lipophilic statins, 81 hydrophilic statins (27.6\%), and $30(10.2 \%)$ a combination of lipophilic and hydrophilic statins (Table 1). In this cohort, patients with any documented statin use were more likely to be older, postmenopausal, have a higher BMI, have less advanced clinical nodal status, undergo breast conserving surgery, and less likely to be treated with 
neoadjuvant chemotherapy compared to those patients who did not use statins (Table 2).

Median follow-up time was 75.1 months (range 2.4-228-9 months) for all patients and median age was 51 (range 22-98). The median time to any failure (local or distant) was 18.9 months (range (3.8-123.0). There was no significant difference in 5- or 10-year OS, DFS, DMFS, or LRRFS based on any statin use (Table 3). Results were also not significantly different when type of statin use was differentiated (hydrophilic, lipophilic, or both) (not shown). For the entire cohort, statins were not predictive of OS, DMFS, or LRR on either univariate (not shown) or multivariate analysis of factors predictive of outcomes (Table 4). For those patient with a local or distant disease failure, any statin use was associated with improved 5- and 10 -year survival compared to no statin use $(34.7 \%$ and $18.8 \%$ versus $25.6 \%$ and $7.4 \%$, respectively, $\mathrm{p}=0.03$ ).
For those patients without any local or distant disease failure, there was no difference and 5- and 10-year survival based on statin use $(p=0.49)$

Table 1. Frequency and Percentage of Statin Use by Statin Type Among Patients Taking Statins

\begin{tabular}{ll}
\hline Statin Type & No (\%) \\
\hline Lipophilic & \\
Atovastatin & $103(35.2 \%)$ \\
Simvastatin & $55(18.8 \%)$ \\
Lovastatin & $8(2.7 \%)$ \\
Fluvastatin & $2(0.7 \%)$ \\
Combination & $14(4.8 \%)$ \\
Hydrophilic Statins & \\
Pravastatin & $37(12.6 \%)$ \\
Rosuvastatin & $41(14.0 \%)$ \\
Combination & $3(1.0 \%)$ \\
Lipophilic and Hydrophilic Statin & $30(10.2 \%)$ \\
\hline
\end{tabular}

Table 2. Clinicopathologic Characteristics of Patients Based on Statin Use and Lipid Panel Availability

\begin{tabular}{|c|c|c|c|c|c|c|c|}
\hline & \multirow[t]{2}{*}{ All Patients No. (\%) } & \multicolumn{2}{|c|}{ Use of Statins No. (\%) } & \multirow[t]{2}{*}{ p-value } & \multicolumn{2}{|c|}{ Cholesterol/Lipid Panel Completed } & \multirow[t]{2}{*}{ p-value } \\
\hline & & Never & Ever & & No & Yes & \\
\hline & $869(100 \%)$ & $576(66.3 \%)$ & $293(33.7 \%)$ & & $501(57.7 \%)$ & $368(42.3 \%)$ & \\
\hline \multicolumn{8}{|l|}{ Statin Use } \\
\hline No Statin Use & $576(66.3 \%)$ & $576(100.0 \%)$ & $0(0.0 \%)$ & • & $364(72.7 \%)$ & $212(57.6 \%)$ & $<.001$ \\
\hline Hydrophilic Statin & $81(9.3 \%)$ & $0(0.0 \%)$ & $8(27.6 \%)$ & & $32(6.4 \%)$ & $49(13.3 \%)$ & \\
\hline Lipophilic Statin & $182(20.9 \%)$ & $0(0.0 \%)$ & $182(62.1 \%)$ & & $97(19.4 \%)$ & $85(23.1 \%)$ & \\
\hline Hydrophilic \& Lipophilic Statin & $30(3.5 \%)$ & $0(0.0 \%)$ & $30(10.2 \%)$ & & $8(1.6 \%)$ & $22(6.0 \%)$ & \\
\hline \multicolumn{8}{|l|}{ Age } \\
\hline$\leq 35$ years & $7(8.6 \%)$ & $71(12.3 \%)$ & $4(1.4 \%)$ & $<.001$ & $53(10.6 \%)$ & $22(6.0 \%)$ & 0.02 \\
\hline$>35$ & $794(91.4 \%)$ & $505(87.7 \%)$ & $289(98.6 \%)$ & & $448(89.4 \%)$ & $346(94.0 \%)$ & \\
\hline \multicolumn{8}{|l|}{ Race } \\
\hline White & $523(60.2 \%)$ & $348(60.4 \%)$ & $175(59.7 \%)$ & 0.051 & $311(62.1 \%)$ & $212(57.6 \%)$ & 0.326 \\
\hline Black & $180(20.7 \%)$ & $109(18.9 \%)$ & $71(24.2 \%)$ & & $94(18.8 \%)$ & $86(23.4 \%)$ & \\
\hline Hispanic & $119(13.7 \%)$ & $90(15.6 \%)$ & $29(9.9 \%)$ & & $71(14.2 \%)$ & $48(13.0 \%)$ & \\
\hline Other & $47(5.4 \%)$ & $29(5.0 \%)$ & $18(6.1 \%)$ & & $25(5.0 \%)$ & $22(6.0 \%)$ & \\
\hline \multicolumn{8}{|l|}{ Menopausal Status } \\
\hline Premenopausal & $553(63.6 \%)$ & $305(53.0 \%)$ & $248(84.6 \%)$ & $<.001$ & $307(61.3 \%)$ & $246(66.8 \%)$ & 0.101 \\
\hline Postmenopausal & $316(36.4 \%)$ & $271(47.0 \%)$ & $45(15.4 \%)$ & & $194(38.7 \%)$ & $122(33.2 \%)$ & \\
\hline \multicolumn{8}{|l|}{ Body Mass Index } \\
\hline$<25$ & $267(30.7 \%)$ & $200(34.7 \%)$ & $67(22.9 \%)$ & $<.001$ & $169(33.7 \%)$ & $98(26.6 \%)$ & 0.028 \\
\hline $25-29.9$ & $255(29.3 \%)$ & $173(30.0 \%)$ & $82(28.0 \%)$ & & $149(29.7 \%)$ & $106(28.8 \%)$ & \\
\hline$\geq 30$ & $346(39.8 \%)$ & $203(35.2 \%)$ & $143(48.8 \%)$ & & $182(36.3 \%)$ & $164(44.6 \%)$ & \\
\hline Unknown & $1(0.1 \%)$ & $0(0.0 \%)$ & $1(0.3 \%)$ & & $1(0.2 \%)$ & $0(0.0 \%)$ & \\
\hline \multicolumn{8}{|l|}{ Clinical Stage Group } \\
\hline Stage I & $215(24.7 \%)$ & $131(22.7 \%)$ & $84(28.7 \%)$ & 0.142 & $130(25.9 \%)$ & $85(23.1 \%)$ & 0.617 \\
\hline Stage II & $442(50.9 \%)$ & $298(51.7 \%)$ & $144(49.1 \%)$ & & $252(50.3 \%)$ & $190(51.6 \%)$ & \\
\hline Stage III & $212(24.4 \%)$ & $147(25.5 \%)$ & $65(22.2 \%)$ & & $119(23.8 \%)$ & $93(25.3 \%)$ & \\
\hline \multicolumn{8}{|l|}{ Pathological Nodal Status } \\
\hline pNO & $597(68.7 \%)$ & $384(66.7 \%)$ & $213(72.7 \%)$ & 0.187 & $338(67.5 \%)$ & $259(70.4 \%)$ & 0.412 \\
\hline pN1 & $170(19.6 \%)$ & $121(21.0 \%)$ & $49(16.7 \%)$ & & $98(19.6 \%)$ & $72(19.6 \%)$ & \\
\hline pN2-3 & $102(11.7 \%)$ & $71(12.3 \%)$ & $31(10.6 \%)$ & & $65(13.0 \%)$ & $37(10.1 \%)$ & \\
\hline \multicolumn{8}{|l|}{ Adjuvant Radiation } \\
\hline No & $236(27.2 \%)$ & $159(27.6 \%)$ & $77(26.3 \%)$ & 0.678 & $143(28.5 \%)$ & $93(25.3 \%)$ & 0.316 \\
\hline Yes & $633(72.8 \%)$ & $417(72.4 \%)$ & $216(73.7 \%)$ & & 358 (71.5\%) & $275(74.7 \%)$ & \\
\hline \multicolumn{8}{|l|}{ Definitive Breast Surgery } \\
\hline BCS & $428(49.3 \%)$ & $262(45.5 \%)$ & $166(56.7 \%)$ & 0.007 & $242(48.3 \%)$ & $186(50.5 \%)$ & 0.512 \\
\hline Mastectomy & $436(50.2 \%)$ & $310(53.8 \%)$ & $126(43.0 \%)$ & & $257(51.3 \%)$ & $179(48.6 \%)$ & \\
\hline ALND Alone & $5(0.6 \%)$ & $4(0.7 \%)$ & $1(0.3 \%)$ & & $2(0.4 \%)$ & $3(0.8 \%)$ & \\
\hline \multicolumn{8}{|l|}{ Chemotherapy } \\
\hline Neoadjuvant & $431(49.6 \%)$ & $301(52.3 \%)$ & $130(44.4 \%)$ & 0.014 & $250(49.9 \%)$ & $181(49.2 \%)$ & 0.295 \\
\hline Adjuvant & $329(37.9 \%)$ & $198(34.4 \%)$ & $131(44.7 \%)$ & & $197(39.3 \%)$ & $132(35.9 \%)$ & \\
\hline Neoadjuvant and Adjuvant & $45(5.2 \%)$ & $35(6.1 \%)$ & $10(3.4 \%)$ & & $23(4.6 \%)$ & $22(6.0 \%)$ & \\
\hline None & $64(7.4 \%)$ & $42(7.3 \%)$ & $22(7.5 \%)$ & & $31(6.2 \%)$ & $33(9.0 \%)$ & \\
\hline \multicolumn{8}{|l|}{ Follow-Up Time (months) } \\
\hline Median (range) & $\begin{array}{l}75.1 \\
(2.4-228.9)\end{array}$ & $\begin{array}{l}72.3 \\
(2.4-205.5)\end{array}$ & $\begin{array}{l}81.4 \\
(9.2-228.9)\end{array}$ & 0.014 & $\begin{array}{l}75 \\
(3.5-228.9)\end{array}$ & $\begin{array}{l}75.3 \\
(2.4-201.9)\end{array}$ & 0.266 \\
\hline
\end{tabular}

$\mathrm{BCS}=$ breast conserving surgery, $\mathrm{ALND}=$ axillary lymph node dissection 
We then restricted analysis to the cohort of 368 patients for whom a 5 -value complete lipid panel was available. In this cohort, median follow-up time was 75.3 months (range 2.4-201.9 months). Patients in this cohort were more likely to use statins, be $>35$ years of age at diagnosis, and have a higher BMI, but had no apparent difference with regards to tumor and treatment characteristics. 5- and 10-year OS, DFS, DMFS, and LRRFS based on any statin use within this cohort demonstrated improved OS with any statin use, with a trend of improved DMFS; no difference was seen for LRRFS. Although not significant, there was a trend of improved 5-/10-year OS and DMFS for patients taking lipophilic statins or a combination of hydrophilic and lipophilic statins compared to those only taking hydrophilic statins (both $\mathrm{p}=.052$ ). Neither individual laboratory values for total cholesterol, HDL, LDL, VLDL nor triglycerides predicted for outcomes (not shown). The relative risk of breast cancer recurrence and breast cancer-related death for both the entire cohort and the cohort of patients with lipid values are shown in Table 4.

Multivariable analysis of factors predictive of OS among those patients with a full lipid panel, controlling for total cholesterol, HDL, LDL, VLDL, triglyceride values was performed, also including clinical stage group, pathological nodal status, histology, and use of chemotherapy. In this model, any statin use was predictive of OS (HR .10, $p=.026$, 95\% CI 0.01-0.76) (Table 5). There was a trend for any statin use to predict outcomes for DMFS, which was not statistically significant (HR 0.14, $\mathrm{p}=0.094,95 \%$ CI 0.01-1.40), and no association of statins in this cohort on LRRFS.

Table 3. 5- and 10-year Survival Outcomes by Statin Use

\begin{tabular}{|c|c|c|c|c|c|c|}
\hline & \multicolumn{3}{|c|}{ Entire Cohort } & \multicolumn{3}{|c|}{$\begin{array}{c}\text { Patients with Complete Lipid } \\
\text { Panel }\end{array}$} \\
\hline & 5-year & 10-year & p-value & 5-year & 10-year & p-value \\
\hline \multicolumn{7}{|c|}{ Overall Survival by Clinical Stage } \\
\hline I & $94.8 \%$ & $87.9 \%$ & $<0.001$ & $98.8 \%$ & $89.7 \%$ & $<.001$ \\
\hline II & $87.4 \%$ & $78.7 \%$ & & $89.2 \%$ & $77.9 \%$ & \\
\hline III & $59.4 \%$ & $54.0 \%$ & & $64.0 \%$ & $55.6 \%$ & \\
\hline \multicolumn{7}{|c|}{ Overall Survival } \\
\hline Statin & $84.7 \%$ & $79.1 \%$ & 0.150 & $89.6 \%$ & $82.8 \%$ & 0.026 \\
\hline No Statin & $81.3 \%$ & $72.8 \%$ & & $81.9 \%$ & $69.0 \%$ & \\
\hline \multicolumn{7}{|c|}{ Disease Free Survival } \\
\hline Statin & $83.1 \%$ & $80.1 \%$ & 0.199 & $84.2 \%$ & $81.6 \%$ & 0.051 \\
\hline No Statin & $78.9 \%$ & $76.8 \%$ & & $76.3 \%$ & $72.4 \%$ & \\
\hline \multicolumn{7}{|c|}{ Distant Metastasis Free Survival } \\
\hline Statin & $85.2 \%$ & $83.3 \%$ & 0.053 & $86.8 \%$ & $85.1 \%$ & 0.014 \\
\hline No Statin & $80.0 \%$ & $77.6 \%$ & & $77.7 \%$ & $74.0 \%$ & \\
\hline \multicolumn{7}{|c|}{ Local-Regional Recurrence Free Survival } \\
\hline Statin & $93.4 \%$ & $91.1 \%$ & 0.766 & $95.3 \%$ & $93.3 \%$ & 0.713 \\
\hline No Statin & $93.1 \%$ & $92.5 \%$ & & $93.4 \%$ & $92.7 \%$ & \\
\hline
\end{tabular}

Table 4. Data Published to Date Regarding Breast Cancer Outcomes and Statin Use

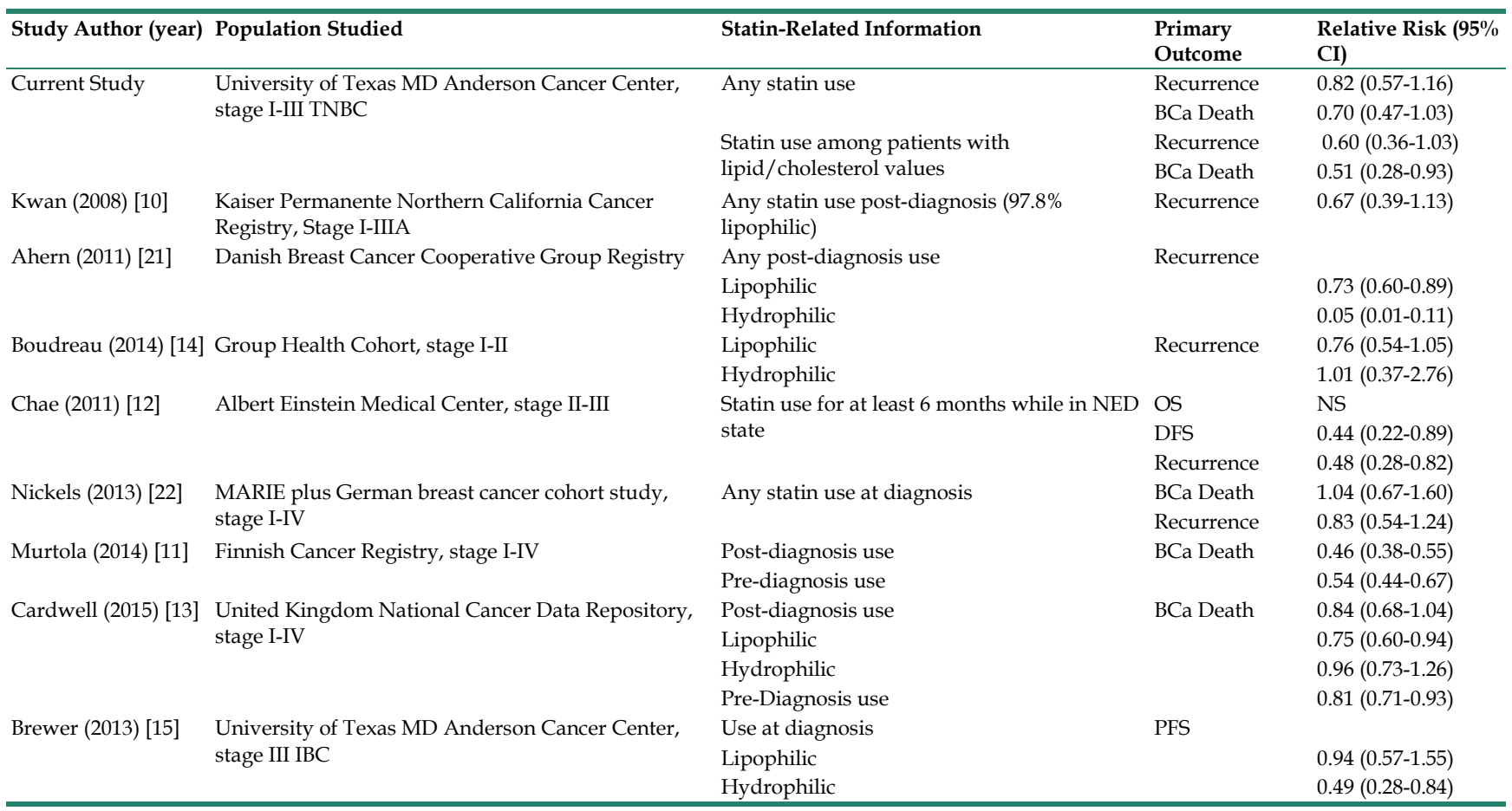

Abbreviations: TNBC: triple negative breast cancer, OS: overall survival, DFS: disease free survival, BCa: Breast 
Table 5. Multivariable Cox Analysis of Factors Predictive of Clinical Outcomes for All Patients and those with a Lipid Panel

\begin{tabular}{|c|c|c|c|c|}
\hline \multirow[t]{2}{*}{ Covariates } & \multicolumn{2}{|l|}{ All Patients } & \multicolumn{2}{|c|}{$\begin{array}{l}\text { Patients with Lipid } \\
\text { Panel Values, Which Are } \\
\text { Added to the } \\
\text { Multivariable Model }\end{array}$} \\
\hline & HR (95\% CI) & p-value & HR $(95 \%$ CI) & p-value \\
\hline \multicolumn{5}{|l|}{ Overall Survival } \\
\hline Statin Use (ref: no) & $0.74(0.20-2.77)$ & 0.656 & $0.10(0.01-0.76)$ & 0.026 \\
\hline BMI (continuous) & $0.99(0.96-1.02)$ & 0.458 & $0.94(0.89-0.99)$ & 0.013 \\
\hline Age $>35$ yrs & $0.71(0.45-1.14)$ & 0.157 & $0.44(0.21-0.93)$ & 0.032 \\
\hline \multicolumn{5}{|c|}{ Distant Metastasis Free Survival } \\
\hline Statin Use (ref: no) & $1.10(0.24-4.92)$ & 0.903 & $0.14(0.01-1.40)$ & 0.094 \\
\hline BMI (continuous) & $0.99(0.96-1.02)$ & 0.641 & $0.93(0.88-0.98)$ & 0.006 \\
\hline Age $>35$ yrs & $0.57(0.36-0.89)$ & 0.015 & $0.37(0.18-0.79)$ & 0.010 \\
\hline \multicolumn{5}{|c|}{ Local-Regional Relapse Free Survival } \\
\hline Statin Use (ref: no) & $2.64(0.28-25.12)$ & 0.398 & $0.10(0.00-3.51)$ & 0.203 \\
\hline BMI (continuous) & $1.01(0.96-1.06)$ & 0.789 & $0.93(0.84-1.03)$ & 0.151 \\
\hline Age $>35$ yrs & $1.26(0.45-3.58)$ & 0.660 & $1.30(0.16-10.42)$ & 0.802 \\
\hline
\end{tabular}

\section{Discussion}

Our study demonstrated that the interaction of any statin use with clinical outcomes in triple negative breast cancer is not yet fully understood. Statin use did not predict for survival or recurrence outcomes in a large unselected cohort of patients with TNBC. However, among a subset of patients for whom cholesterol and triglyceride values could be controlled for, any statin use predicted for improved overall survival. There also appeared to be an association of statins with survival among patients who experienced a local-regional or distant metastatic failure.

Those patients who had lipid values available were more likely to take statins, have an elevated BMI, and be older than 35 years of age. However, there were no apparent tumor-specific differences with regards to stage or treatments delivered between those patients with versus those without lipid panels. Statin use, BMI and age at diagnosis all remained significant on multivariable analysis controlling for lipid values and tumor and cancer treatment-specific factors, highlighting the importance of each of these factors on breast cancer clinical outcomes. Indeed, any statin use had more of a protective effect on survival outcomes than either BMI or older age at diagnosis.

The absence of effect in those without lipid panels could imply spurious results, or great complexity of the biologic processes involved, necessitating understanding of all the potential variables involved. Alternatively, it may imply an unmeasured correlation between the underlying causes of hypertension, familial dyslipidemia and other factors that prompt lipid panels and sensitivity to statins. Interestingly, Cai et al recently published that $8 \mathrm{p}$ loss of heterozygosity $(\mathrm{LOH})$, a common deletion in breast cancer across all subtypes, is significantly associated with altered lipid biosynthesis genes in TCGA patient data and alters lipid composition but not cholesterol levels in experimental models [18]. They reported that $8 \mathrm{p} \mathrm{LOH}$ cells are more resistant to statin and other standard treatments. Kullo et al examining 691 non-Hispanic white individuals ascertained through sibships with two or more individuals diagnosed with hypertension before age 60 reported evidence of LDL particle size linkage to microsatellite marker loci on 8p [19]. In an earlier sibpair analysis, Knoblauch et al reported significant linkage for HDL-C and TGs, with D8S261 on chromosome $8 \mathrm{p}$ that they find to be related to macrophage scavenger receptor genes rather than the lipoprotein lipase gene also located in this region [20]. The relationship between $8 p$ microsatellite instability and $\mathrm{LOH}$ in tumor and normal cells as well as to the impact of lipids on both cell types warrants further investigation.

One hypothesis for a meaningful, greater impact of any statin use on overall survival than disease free survival among those with lipid panels is that patients with recurrence who had been taking statins lived longer either due to more indolent nature of the recurrence influenced by the use of statins or due to a greater sensitivity of the disease to therapy at the time of recurrence influenced by statins. The former suggests a selective pressure mechanism and imply that giving statins at the time of relapse would not improve outcome as the biology of the recurrent cells has already been determined. The latter would imply adding statins to metastatic regimens could have some benefit. Of course potentially both issues may be true to some degree. Consistent with the hypothesis that statins promote drug sensitivity and thus outcome after recurrence rather than impacting recurrence itself, Cai et al report that $8 \mathrm{pLOH}$ does not impact tumorigenesis, but only treatment sensitivity [18]. If true, this might imply that trials testing statins that exclude patients who are already on statins may be excluding the population that benefits. Importantly, the work by Cai et al provide an easily testable biomarker for the influence of statins that could not be assessed in this cohort.

Statin use has been found to be associated with differential outcomes with regards to breast cancer recurrence (Table 4). A retrospective cohort study of 1,945 women diagnosed with early stage breast cancer between 1997 and 2000 in the Kaiser Permanente Northern California Cancer Registry examined statin use and breast cancer recurrences [10]. The vast majority of patients studied $(97.8 \%)$ used lipophilic statins for a mean duration of 1.96 years. The risk of recurrence decreased with increasing duration of 
statin use, and there was a trend of an association between statin use after breast cancer diagnosis and decreased risk of recurrence. A population-based cohort study in Finland that included 31,236 cases of breast cancer demonstrated that breast cancer-specific mortality was approximately halved by the use of either pre-diagnostic or post-diagnostic statin use, with a dose-dependent mortality benefit seen for those taking statins prior to diagnosis [11]. Other studies have shown weak or mixed associations of statin use on breast cancer-specific mortality and breast cancer recurrence [12-14]. Our results highlight the potentially complex interaction between statin use, lipids, body mass index, metabolic syndrome, and possibly other heretofore unidentified factors. It bears mentioning that in addition to a potential association between statin use and breast cancer specific outcomes, statin use in and of itself might impact overall survival in a breast cancer cohort secondary to cardiovascular endpoints.

The type of statin used (hydrophilic, lipophilic, or both) was not predictive of outcomes in our study, but this may have been limited by our study size or may indicate a receptor subtype specificity to this effect. A large population-based cohort study in Denmark found that use of lipophilic statins was associated with a decreased risk of breast cancer recurrence, whereas hydrophilic statin use had no such association [21]. In this study, any statin use had a preferential impact on estrogen receptor positive but not estrogen receptor negative tumors and impacted local-regional and contralateral breast recurrences. At our institution, hydrophilic statin use has previously been associated with improved progression free survival among patients with inflammatory breast cancer [15]. Nevertheless, the broadest case for statin benefit across the breast cancer literature is thus far for the lipophilic statin, simvastatin [22]. This may reflect distinct differences in IBC cells cholesterol levels and equilibrium [23].

Although the benefit of statin was only identified among those with lipid profiles available for inclusion in the analysis, no specific lipoprotein was associated with outcome. We had previously seen HDL superseded statin use in IBC [16]. A recent prospective analysis of lipoproteins in 56 women with predominantly ER+ breast cancer demonstrated some preliminary associations between HDL fractions and progesterone expression as well as ki-67 [24]. This may implicate a further degree of complexity in determining the role of statins.

Strengths of our study include inclusion of a large number of patients with TNBC with long follow-up. Our dataset also includes detailed information on body mass index as well as information on location of recurrence (distant metastases and/or local regional recurrence), thereby enabling conclusions on potential differential modes of cholesterol-mediated spread of breast cancer. Further we were able to extract the lipid levels where available to provide additional information on circulating lipoproteins.

Our study contains limitations inherent in a retrospective chart review. In particular, those patients for whom a five-value lipid panel was performed at our institution may have represented a biased cohort, as these laboratory values are not routinely checked at our cancer center. Lipid panels were typically checked for those patients who required general internal medicine or cardiology care while they were undergoing cancer care. We recorded data based on any statin use documented from the time of first presentation at our institution through last follow-up. Information on duration of statin use was not available, nor was medication compliance. Prior studies have highlighted that duration of statin use in and of itself may be predictive of the risk of breast cancer recurrence [10]. It may be that statin use in and of itself could be a marker of patient engagement and/or compliance with medical recommendations. Our study was limited by a lack of information about use of aspirin, metformin, or other similar agents, which are being investigated in ongoing randomized trials to understand their impact on breast cancer recurrence. that may also have antineoplastic properties in the setting of breast cancer [25] [26].

\section{Conclusions}

Any statin use among patients with TNBC is not associated with improved OS. However, when controlling for other relevant clinical factors including serum lipids, statins may impact oncologic outcomes. Given the complex interplay of obesity, statin use, lipid levels, comorbidities, and age, we recommend prospective assessment of these values on a clinical trial to better define the value of adding statins as a potential treatment to patients with TNBC.

\section{Competing Interests}

M.C. Stauder is a consultant for the M.D. Anderson Physician's Network. The other authors have no conflicts of interest to disclose.

\section{References}

1. Foulkes WD, Smith IE, Reis-Filho JS. Triple-negative breast cancer. N Engl J Med. 2010;363: 1938-48

2. Dent R, Trudeau M, Pritchard KI, Hanna WM, Kahn HK, Sawka CA, et al. Triple-negative breast cancer: clinical features and patterns of recurrence. Clin Cancer Res. 2007; 13: 4429-34.

3. Le Du F, Eckhardt BL, Lim B, Litton JK, Moulder S, Meric-Bernstam F, et al. Is the future of personalized therapy in triple-negative breast cancer based on molecular subtype? Oncotarget. 2015; 6: 12890-908. 
4. Melhem-Bertrandt A, Chavez-Macgregor M, Lei X, Brown EN, Lee RT, Meric-Bernstam F, et al. Beta-blocker use is associated with improved relapse-free survival in patients with triple-negative breast cancer. J Clin Oncol. 2011; 29: 2645-52.

5. Bayraktar S, Hernadez-Aya LF, Lei X, Meric-Bernstam F, Litton JK, Hsu L, et al. Effect of metformin on survival outcomes in diabetic patients with triple receptor-negative breast cancer. Cancer. 2012; 118: 1202-11.

6. Nelson ER, Chang CY, McDonnell DP. Cholesterol and breast cancer pathophysiology. Trends in endocrinology and metabolism: TEM. 2014; 25: 649-55.

7. Wolfe AR, Debeb BG, Lacerda L, Larson R, Bambhroliya A, Huang X, et al. Simvastatin prevents triple-negative breast cancer metastasis in pre-clinical models through regulation of FOXO3a. Breast Cancer Res Treat. 2015; 154: 495-508.

8. Ginestier C, Monville F, Wicinski J, Cabaud O, Cervera N, Josselin E, et al. Mevalonate metabolism regulates Basal breast cancer stem cells and is a potential therapeutic target. Stem Cells. 2012; 30: 1327-37.

9. Park YH, Jung HH, Ahn JS, Im YH. Statin induces inhibition of triple negative breast cancer (TNBC) cells via PI3K pathway. Biochem Biophys Res Commun. 2013; 439: 275-9.

10. Kwan ML, Habel LA, Flick ED, Quesenberry CP, Caan B. Post-diagnosis statin use and breast cancer recurrence in a prospective cohort study of early stage breast cancer survivors. Breast Cancer Res Treat. 2008; 109: 573-9.

11. Murtola TJ, Visvanathan K, Artama M, Vainio H, Pukkala E. Statin use and breast cancer survival: a nationwide cohort study from Finland. PLoS One. 2014; 9: e110231

12. Chae YK, Valsecchi ME, Kim J, Bianchi AL, Khemasuwan D, Desai A, et al. Reduced risk of breast cancer recurrence in patients using ACE inhibitors, ARBs, and/or statins. Cancer Invest. 2011; 29: 585-93.

13. Cardwell CR, Hicks BM, Hughes C, Murray LJ. Statin use after diagnosis of breast cancer and survival: a population-based cohort study. Epidemiology. 2015; 26: 68-78.

14. Boudreau DM, Yu O, Chubak J, Wirtz HS, Bowles EJ, Fujii M, et al. Comparative safety of cardiovascular medication use and breast cancer outcomes among women with early stage breast cancer. Breast Cancer Res Treat. 2014; 144: 405-16.

15. Brewer TM, Masuda H, Liu DD, Shen Y, Liu P, Iwamoto T, et al. Statin use in primary inflammatory breast cancer: a cohort study. Br J Cancer. 2013; 109: $318-24$.

16. Wolfe AR, Atkinson RL, Reddy JP, Debeb BG, Larson R, Li L, et al. High-density and very-low-density lipoprotein have opposing roles in regulating tumor-initiating cells and sensitivity to radiation in inflammatory breast cancer. Int J Radiat Oncol Biol Phys. 2015; 91: 1072-80.

17. Schachter M. Chemical, pharmacokinetic and pharmacodynamic properties of statins: an update. Fundam Clin Pharmacol. 2005. 19: 117-25.

18. Cai Y, Crowther J, Pastor T, Abbasi Asbagh L, Baietti MF, De Troyer M, et al. Loss of Chromosome 8p Governs Tumor Progression and Drug Response by Altering Lipid Metabolism. Cancer Cell. 2016; 29: 751-66.

19. Kullo IJ, Turner ST, Boerwinkle E, Kardia SL, de Andrade M. A novel quantitative trait locus on chromosome 1 with pleiotropic effects on HDL-cholesterol and LDL particle size in hypertensive sibships. Am J Hypertens. 2005; 18: 1084-90.

20. Knoblauch H, Busjahn A, Munter S, Nagy Z, Faulhaber HD, Schuster H, et al. Heritability analysis of lipids and three gene loci in twins link the macrophage scavenger receptor to HDL cholesterol concentrations. Arterioscler Thromb Vasc Biol. 1997; 17: 2054-60.

21. Ahern TP, Pedersen L, Tarp M, Cronin-Fenton DP, Garne JP, Silliman RA, et al. Statin prescriptions and breast cancer recurrence risk: a Danish nationwide prospective cohort study. J Natl Cancer Inst. 2011; 103: 1461-8.

22. Ahern TP, Lash TL, Damkier P, Christiansen PM, Cronin-Fenton DP. Statins and breast cancer prognosis: evidence and opportunities. Lancet Oncol. 2014; 15: e461-8.

23. Martin BJ, van Golen KL. A comparison of cholesterol uptake and storage in inflammatory and noninflammatory breast cancer cells. Int J Breast Cancer. 2012; 2012: 412581.

24. Flote VG, Vettukattil R, Bathen TF, Egeland T, McTiernan A, Frydenberg H, et al. Lipoprotein subfractions by nuclear magnetic resonance are associated with tumor characteristics in breast cancer. Lipids Health Dis. 2016; 15: 56.

25. Oncology AfCTi. Aspirin in preventing recurrence of cancer in patients with node positive HER2 negative stage II-III bresat cancer after chemotherapy, surgery, and/or radiation therapy. 2016.

26. Group NCT. A phase III randomized trial of metformin vs placebo in early stage breast cancer. 